\title{
Pain Hypersensitivity: A Bio-Psychological Explanation of Chronic Musculoskeletal Pain and Underpinning Theory
}

\author{
Zakir Uddin1,2*, Joy C. MacDermid1,3 \\ ${ }^{1}$ School of Rehabilitation Science, McMaster University, Hamilton, Ontario, Canada \\ ${ }^{2}$ Department of Physical Therapy, College of Health and Welfare, Woosong University, Daejeon, South Korea \\ ${ }^{3}$ Clinical Research Lab, Hand and Upper Limb Centre, St. Joseph's Health Centre, London, Ontario, Canada \\ Email: $\underline{\text { uddinz2@mcmaster.ca, }}$ akkiru@gmail.com
}

Received 15 January 2014; revised 20 February 2014; accepted 17 March 2014

Copyright $(02014$ by authors and Scientific Research Publishing Inc.

This work is licensed under the Creative Commons Attribution International License (CC BY).

http://creativecommons.org/licenses/by/4.0/

(c) (i) Open Access

\begin{abstract}
Hypersensitivity is a phenomenon that has a dual role: adaptive (protective) and maladaptive (pathological) based on different aspects of the pain mechanism. The mechanism of hypersensitivity has not been fully defined. However, it is known that over-excitability (too much sensitivity) of neurons can arise in both peripheral and central components of the nervous system. Pain theories can be useful in helping to explain complex phenomenon like hypersensitivity. The Gate control theory and other more bio-psychological pain models may assist us to understand a mechanism of chronic musculoskeletal pain. This article discusses a mechanism based pain model.
\end{abstract}

\section{Keywords}

Abnormal Pain, Theory, Sensitivity

\section{Introduction}

Human pain systems need to be sensitive to detect potential harm for the body. However, pain systems can become too overly sensitive when innocuous stimuli are perceived as being painful, causing us unnecessary pain without any protective benefit. This phenomenon is known as hypersensitivity. It arises because there is an increase in sensitivity of pain pathways that relay pain messages to the brain. The mechanisms of this sensitization are not yet clear. However, it is known that sensitization (increase in the excitability of neurons) is involved in both peripheral and central nervous system [1] [2].

${ }^{*}$ Corresponding author.

How to cite this paper: Uddin, Z. and MacDermid, J.C. (2014) Pain Hypersensitivity: A Bio-Psychological Explanation of Chronic Musculoskeletal Pain and Underpinning Theory. Pain Studies and Treatment, 2, 31-35.

http://dx.doi.org/10.4236/pst.2014.22007 
In a broader sense, pain can be classified into two fundamental categories: physiological/adaptive (nociceptive) pain and pathological/maladaptive (non-nociceptive) pain [3] [4]. Physiological pain serves an important protective function since it is the mechanism for people removing themselves from harm, or seeking care for health problems. On the other hand, pathological pain has a major negative impact on quality of life in the context of human disease.

Normally, pain is produced only by noxious stimuli (commonly known as painful/intense stimuli) that are damaging to tissue (potentially or actually) [3]. This adaptive/physiological pain is mediated by a special neural process of encoding noxious stimuli (nociception) and designed to respond only to such noxious/painful stimuli in both peripheral and central neurons [5]. Adaptive pain is an essential early warning protective device of the body that helps to prevent harm in the dangerous environment. To do this, pain sensation needs to be unpleasant as an alarming signal to protect body tissues.

Pathological/maladaptive pain occurs in response to persistent tissue injury, damage to the nervous system itself (neuropathic pain) and changes in the normal neural function (functional pain) [1]-[4]. It features pain without apparent peripheral stimulus but often with manifest hypersensitivity. Clinically, we can observe two distinct abnormal pain responses (two features of hypersensitivity): allodynia and hyperalgesia (see Table 1) [5] [6]. It is clinically important to assess or quantify these two types of hypersensitivity to optimize therapy.

The good role of pain hypersensitivity is that it helps healing after an injury by minimizing contact or movement of the injured tissue until repair is complete (an adaptive response of the healing process). On the other hand, the bad role is that it may persist after the healing process of injury or even in the absence of any injury. In this case, pain provides us with no benefits (neither protective nor healing). It is a manifestation of pathological change in the nervous system and clinically it is known as chronic pain (a disease of the nervous system itself) [2] [7]. This article discusses a mechanism based bio-psychological pain model (i.e. pain hypersensitivity/sensitization) to explain chronic musculoskeletal pain.

\section{The Need of a Theoretical Frame}

It is very difficult to explain the complex mechanism of chronic pain without a theoretical construct of pain mechanism. The Gate Control Theory (GCT) can explanation pain hypersensitivity and it may assist us to understand a mechanism of chronic musculoskeletal pain. In 1965, Melzack and Wall proposed that a gate system exists in the dorsal horn of spinal cord and the gate regulating pain by a dynamic facilitatory and inhibitory mechanism [8]. There are four basic structural components of the gate control theory (Figure 1): small fibers (A-delta and C fiber), large fiber (A-beta), substantia gelatinosa (SG, lamina-II of dorsal horn), and the hypothetical first central transmission cell ( $\mathrm{T}$ cell). The theory describes the relationship between these structures. The large and small fibers project toward the SG and T cell. An inhibitory effect on pain processing is increased in SG by large fibers and decreased by small fibers. A diagrammatic representation of the GCT (Figure 1) shows the gate control system in the dorsal horn receiving feedback from the central control, and reflecting the central inhibitory influence of pain [8]. The central inhibitory influence plays a major role in hypersensitivity and in chronic pain.

\section{Hypersensitivity Model within the Theory}

The GCT has been used to explain the role of the spinal cord and brain in the perception of both acute and chronic pain [9]. The gate control system models how peripheral stimuli interact to modulate nociception before it evokes pain perception. Specifically, it explains the role of the A-beta fiber, nociceptors, and the dorsal horn

Table 1. Two common Hypersensitivity phenomena: Allodynia and Hyperalgesia, and their differences [5] [20].

\begin{tabular}{|c|c|c|}
\hline Topic & Allodynia & Hyperalgesia \\
\hline IASP Definition [20] & $\begin{array}{l}\text { Pain due to a stimulus that does } \\
\text { not normally provoke pain }\end{array}$ & $\begin{array}{l}\text { Increased pain from a stimulus that } \\
\text { normally provokes pain }\end{array}$ \\
\hline Pain mechanism & Lowered threshold & Increased response \\
\hline Stimulus and response mode & Differ & Same \\
\hline
\end{tabular}

IASP: International Association for the Study of Pain. 


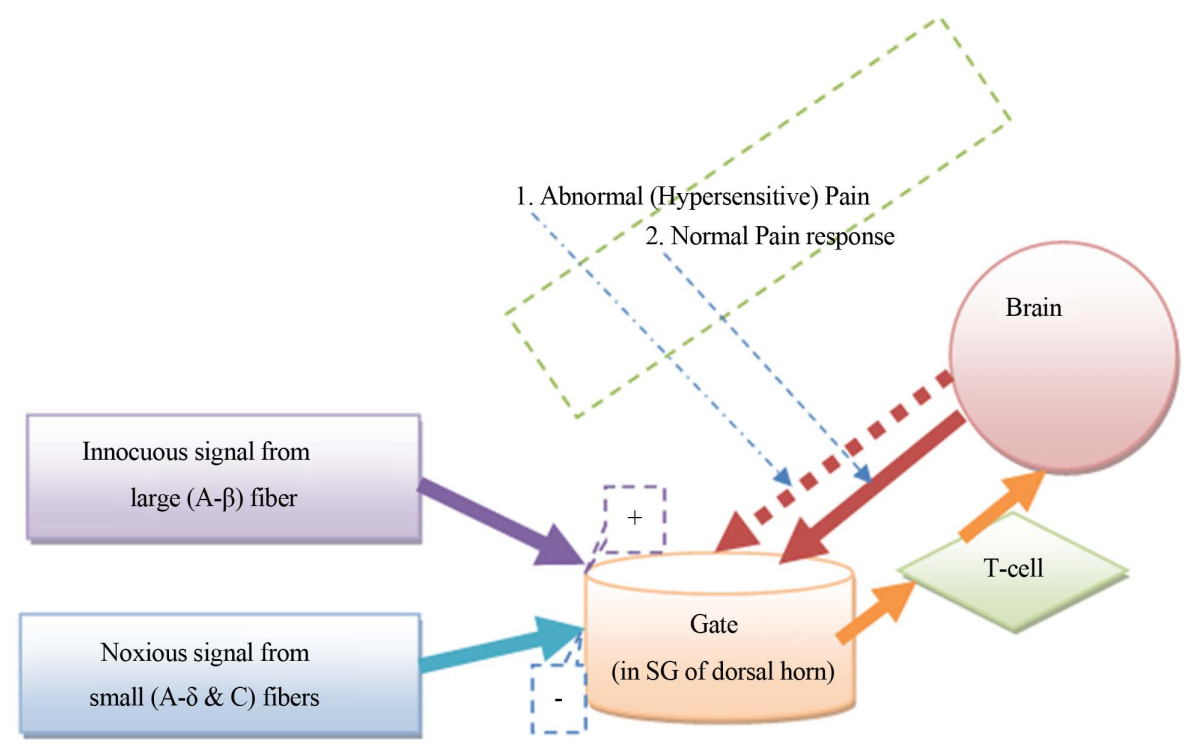

Figure 1. Gate control theory, [8]: Components and basic link of gate mechanism with normal and abnormal (hypersensitive) pain response. SG = Substantia Gelatinosa; $\mathrm{T}$-cell $=1$ st central Transmission cell. Hypersensitivity manifestation is considered as an abnormal central inhibitory control (interrupted top down control) at the gate controlling mechanism (interrupted arrow pathway).

in the central transmission of pain. It reflects neurophysiologic mechanisms like, central summation of pain, and sensory input into the control of pain. When it was introduced, the GCT moved thinking about how pain is "generated" away from the periphery into the spinal cord, with central summation of pain perception and sensory input control becoming more considered when thinking about pain perception arises. Fundamentally, the GCT focuses on pain modulation in the dorsal horn by two opposing controlling mechanisms (facilitation and inhibition). In chronic pain, hypersensitivity thought to develop from changes to the physiological inhibitory component of the GCT. Hypersensitivity manifestation in GCT is considered as an abnormal central inhibitory control at the gate controlling mechanism (Figure 1).

The GCT explains pain regulation in a way that can account for normal and hypersensitive (abnormal) pain responses. The GCT hypothesized that nociception (pain sensation) is dynamically regulated in the dorsal horn of the spinal cord; leading to an increase or decrease pain sensitivity [10]. Disturbance in the inhibitory mechanism has been implicated as one of the major causes of hypersensitivity [6] [11]-[14]. Hypersensitivity reflects imbalance within the opposing neural activities in the gate control.

\section{Influence of the Theory in Pain and Rehabilitation Research}

GCT has had a major influence on the direction of pain research and led to many new findings. This was illustrated in a meta-trend analysis of pain research from 1975 to 2007 demonstrated that the original GCT paper [8] was the top most cited article [15]. That study reviewed 4525 research papers published in "Pain" (the official journal of the International Association for the Study of Pain) and indicates that GCT is the predominant theory in pain research. The meta-trend analysis showed chronic pain was the most studied clinical condition, and an increasing trend for research addressing hypersensitivity.

Within pain interventional research, rehabilitation has interpreted electrotherapy interventions like TENS (Transcutaneous Electrical Stimulation) to work using a GCT lens. The importance of this is evident in a recent bibliometric analysis of research studies on all rehabilitation treatment interventions [16]. This analysis demonstrated that physical agent modalities were the most investigated topic [16]. The study analyzed the 2519 published treatment articles from 1980 to 2009 in "Physical Therapy" journal. This reflects the predominance of physical therapy research that relates to interventions that depend on the conceptual premise of closing the gate of GCT using A-beta fiber pain inhibition. 


\section{The Theory Used in Rehabilitation Practice}

GCT has been used in rehabilitation practice over the last four decades. One of the propositions of GCT explains the consequences of large fiber stimulation and its feedback on pain modulation. Rehabilitation uses that principle to achieve pain control through manipulation of the fast conductive large fibers [8]. Physical modalities and some other therapies are developed based on principles of achieving pain relief via large fibers stimulation. For example, touch can directly affect these large fibers. GCT has an influential role in our understanding of the therapeutics of electro-analgesia. Although electric stimulation has been used to treat pain since the ancient time of Aristotle, [17] some thought this practice was quackery. GCT provided the scientific foundation for the use of electro stimulation. After the publication of Wall and Sweet, [18] electrical stimulation for pain relief was accepted by the medical community. Evidence on the chemical basis for electrical nerve stimulation was discovered later when endogenous opioids supported the use of electrical stimulations, where GCT used as an explanatory model [19].

The rise of GCT has coincided with an increase recognition of the importance of understanding mechanism and theory in the therapy profession. As such, the theory has been integrated in pain curriculum for many physical therapists; and can form the basis of understanding how different rehabilitation intervention might affect pain. Clinically, many pain related phenomena are poorly understood, but rehabilitation professionals can use a GCT rationale to explain pain from unknown origin or nonorganic diseases (i.e. phenomena like referred pain, pain hypersensitivity, etc.).

Since GCT suggests that pain perception and response is triggered by both sensory feedback and central integration via the spinal cord, this can give us "levers" to alter the perception of pain via sensory input. As a physical therapy example, desensitization uses graded exposure to different sensory inputs to modify the pain input, which would lead to reduce pain sensitivity by the inhibitory mechanism of the GCT. Many interventions use in rehabilitation include hand-ons interventions (like manual therapy), graded activity or exercise result in sensory inputs into the "gate" in a way that facilitates better internal pain control. Knowing the theory can help rehabilitation professionals extend their interventions into other innovative ways to alter pain.

\section{Conclusion}

Pain in human is a multidimensional-complex perception that causes a large burden to individuals and society. Various theories of pain have been proposed over the centuries, although few are universally accepted or explain all pain phenomena. The "gate control theory" is one of them or a predominant and long-lasting model used to explain pain and has been very useful in explaining pain phenomena including hypersensitivity. The theory helps to explain the existence of hypersensitivity and some of the rehabilitation interventions that can be used to counteract it.

\section{Conflict of Interests}

We, the authors of the manuscript, do not have a direct/indirect financial relation with the commercial/noncommercial identities mentioned in the paper that might lead to a conflict of interests.

\section{Acknowledgements}

ZU was supported by the McMaster University School of Rehabilitation Science Graduate Scholarship, Canadian National Graduate Scholarship in Rehabilitation Science and Islamic Development Bank Merit scholarship for PhD study.

\section{References}

[1] Woolf, C.J. (2011) Central Sensitization: Implications for the Diagnosis and Treatment of Pain. Pain, 152, S2-S15.

[2] Woolf, C.J. and Salter, M.W. (2000) Neuronal Plasticity: Increasing the Gain in Pain. Science, 288, 1765-1769.

[3] Woolf, C.J. (2010) What Is This Thing Called Pain? Journal of Clinical Investment, 120, 3742-3744.

[4] Woolf, C.J., Bennett, G., Doherty, M., et al. (1998) Towards a Mechanism-Based Classification of Pain? Pain, 77, 227-229.

[5] Merskey, H. and Bogduk, N. (1994) Classification of Chronic Pain. IASP Press, Seattle. 
[6] Sandkühler, J. (2009) Models and Mechanisms of Hyperalgesia and Allodynia. Physiological Review, 89, 707-758.

[7] Henry, J.L. (2008) The Need for Knowledge Translation in Chronic Pain. Pain Research Management, 13, 465-476.

[8] Melzack, R. and Wall, P.D. (1965) Pain Mechanisms: A New Theory. Science, 150, 971-979.

[9] Melzack, R. and Wall, P.D. (1988) The Challenge of Pain. Basic Books, New York.

[10] Mallen, C., Peat, G., Thomas, E. and Croft, P. (2007) Prognostic Factors for Musculoskeletal Pain in Primary Care: A Systematic Review. British Journal of General Practice, 57, 655-661.

[11] Sandkühler, J. (2009) The Roles of Inhibition for the Generation and Amplification of Pain. In: Castro-Lopes, J., Ed., Current Topics in Pain, IASP Press, Seattle, 53-71.

[12] Kuner, R. (2010) Central Mechanisms of Pathological Pain. Natural Medicine, 16, 1258-1266.

[13] Woolf, C.J. (2004) Pain: Moving from Symptom Control toward Mechanism-Specific Pharmacologic Management. Annals of International Medicine, 140, 441-451.

[14] Curatolo, M., Arendt-Nielsen, L. and Petersen-Felix, S. (2006) Central Hypersensitivity in Chronic Pain: Mechanisms and Clinical Implications. Physical Medicine \& Rehabilitation Clinics of North America, 17, 287-302.

[15] Mogil, J.S., Simmonds, K. and Simmonds, M.J. (2009) Pain Research from 1975 to 2007: A Categorical and Bibliometric Meta-Trend Analysis of Every Research Paper Published in the Journal. Pain, 142, 48-58.

[16] Coronado, R.A., Riddle, D.L., Wurtzel, W.A., et al. (2011) Bibliometric Analysis of Articles Published from 1980 to 2009 in Physical Therapy. Journal of the American Physical Therapy Association. Physical Therapy, 91, 642-655.

[17] Kane, K. and Taub, A. (1975) A History of Local Electrical Analgesia. Pain, 1, 125-138.

[18] Wall, P.D. and Sweet, W.H. (1967) Temporary Abolition of Pain in Man. Science, 155, 108-109.

[19] Sluka, K.A. and Walsh, D. (2003) Transcutaneous Electrical Nerve Stimulation: Basic Science Mechanisms and Clinical Effectiveness. Journal of Pain, 4, 109-121.

[20] IASP Taxonomy (2011) Pain Terms. http://www.iasp-pain.org/Content/NavigationMenu/GeneralResourceLinks/PainDefinitions/

\section{Abbreviation}

GCT $=$ Gate Control Theory

SG = Substantia Gelatinosa

$\mathrm{T}$ cell $=$ Transmission cell 